\title{
Electron Heating and Cosmic Rays at a Supernova Shock from Chandra X-ray Observations of E0102.2-7219
}

\author{
John P. Hughes ${ }^{1,2}$, Cara E. Rakowski ${ }^{1,2}$, and Anne Decourchelle ${ }^{2}$
}

\begin{abstract}
In this Letter we use the unprecedented spatial resolution of the Chandra X-Ray Observatory to carry out, for the first time, a measurement of the post-shock electron temperature and proper motion of a young SNR, specifically to address questions about the post-shock partition of energy among electrons, ions, and cosmic rays. The expansion rate, $0.100 \% \pm 0.025 \% \mathrm{yr}^{-1}$, and inferred age, $\sim 1000 \mathrm{yr}$, of E0102.2-7219, from a comparison of X-ray observations spanning 20 years, are fully consistent with previous estimates based on studies of high velocity oxygen-rich optical filaments in the remnant. With a radius of $6.4 \mathrm{pc}$ for the blast wave estimated from the Chandra image, our expansion rate implies a blast wave velocity of $\sim 6000 \mathrm{~km} \mathrm{~s}^{-1}$ and a range of electron temperatures $2.5-45 \mathrm{keV}$, dependent on the degree of collisionless electron heating. Analysis of the Chandra ACIS spectrum of the immediate post-shock region reveals a thermal plasma with abundances and column density typical of the Small Magellanic Cloud and an electron temperature of $0.4-1 \mathrm{keV}$. The measured electron temperature is significantly lower than the plausible range above, which can only be reconciled if we assume that a significant fraction of the shock energy, rather than contributing to the heating of the post-shock electrons and ions, has gone into generating cosmic rays.
\end{abstract}

Subject headings: cosmic rays — ISM: individual (E0102.2-7219) — shock waves supernova remnants - X-rays: ISM

\section{INTRODUCTION}

Supernova remnants (SNRs) are among that rare class of astronomical objects whose dynamical evolution can be probed directly through both radial velocity and proper motion measurements. Together such studies provide important constraints on the evolution of young SNRs and the structure of the ejecta and ambient medium. Furthermore, when its distance is

\footnotetext{
${ }^{1}$ Department of Physics and Astronomy, Rutgers The State University of New Jersey, 136 Frelinghuysen Road, Piscataway NJ 08854-8019; E-mail: jph@physics.rutgers.edu and rakowski@physics.rutgers.edu

${ }^{2}$ Service d'Astrophysique, L'Orme des Merisiers, CEA-Saclay, 91191 Gif-sur-Yvette Cedex France
} 
known, measurement of a remnant's proper motion is equivalent to a measurement of the velocity of the supernova blast wave. This fundamental quantity determines the total energy available to the remnant for partition into bulk kinetic energy of the ambient medium, shock-heated electrons and ions, and relativistic particles, i.e., cosmic rays (see Chevalier 1983; Decourchelle, Ellison, \& Ballet 2000). Conservation laws provide some constraints on how the shock energy is divided up into the several forms, but some issues, such as the fraction of shock energy that goes into cosmic rays and the extent of collisionless heating of the electrons, are still theoretically uncertain and need to be determined observationally.

The brightest X-ray supernova remnant (SNR) in the Small Magellanic Cloud (SMC), E0102.2-7219, is dominated by line emission from highly ionized atoms of $\mathrm{O}, \mathrm{Ne}$, and $\mathrm{Mg}$ (Hayashi et al. 1994; Gaetz et al. 2000) and its X-ray morphology is that of a strongly limb-brightened shell, roughly $40^{\prime \prime}$ in diameter (Hughes 1988). Optical filaments in the remnant are also rich in $\mathrm{O}$ and $\mathrm{Ne}$, extend over a diameter of $24^{\prime \prime}$ (Dopita et al. 1981), and display radial velocities that span a range of $6500 \mathrm{~km} \mathrm{~s}^{-1}$ (Tuohy \& Dopita 1983). At the distance to the SMC (60 kpc, e.g., van den Bergh 2000), the proper motion of the optical filaments should be 0 ". $011 \mathrm{yr}^{-1}$ or an expansion rate of $0.092 \% \mathrm{yr}^{-1}$. Herein we determine the X-ray expansion rate, equivalent to the velocity of the shock, to obtain an estimate of the total shock energy. We then compare this to the electron temperature in the immediate post-shock region, through fits to spectra isolated by Chandra's superb spatial resolution, to determine the fraction of energy in shock-heated electrons.

\section{EXPANSION RATE}

Prior to the launch of Chandra the highest spatial resolution images of E0102.2-7219 were those taken by the Einstein and ROSAT High Resolution Imagers, hereafter EHRI and RHRI. During Orbital Activation and Check-out of Chandra, E0102.2-7219 was observed by the back-side illuminated chip (S3) of the ACIS-S instrument (Garmire 1997). A processed event file (id no. 1231 created on 1999-09-07T18:39:11) was obtained from the Chandra X-ray Center and analysis was carried out using standard astronomical software. For spectral analysis (§3) the events were gain-corrected using the appropriate gain map for a focal plane temperature of -100 C. Events were time filtered to remove intervals of high background as well as frames with bad or no aspect. Dead and flickering pixels were also removed. An ephemeris of all three observations is given in Table 1.

The EHRI and RHRI each made broad-band X-ray images, but in slightly different spectral bands. The possibility of spectral variations with position has been the main factor limiting the widespread use of EHRI and RHRI images for carrying out precise proper motion studies of X-ray SNRs. Now, however, the Chandra ACIS-S data provide a spectral dimension that can be used to take full advantage of the long time baseline for proper motion studies set by the earlier observations. 
In our approach we slice the ACIS-S data into a large number (50-100) of images according to observed photon energy using narrow X-ray spectral bands. These separate images are then multiplied by the ratio of effective area between the EHRI (for example) and the Chandra ACIS-S at the photon energy appropriate to the spectral band of each individual image slice. The final ACIS-S image, corrected to the EHRI band in this case, is then the sum of the image slices. This is done for both of the HRIs. Clearly the accuracy of this procedure depends on how well the various effective area functions are known, an effect we explore below.

The on-orbit $50 \%$ encircled energy radius for the Chandra ACIS-S, $R_{50 \%}=0$ ". 4 (Dewey 1999), is about an order of magnitude smaller than that of the RHRI or EHRI, both of which had $R_{50 \%} \approx 4^{\prime \prime}$ (Giacconi et al. 1979; David et al. 1998). We can therefore ignore the small Chandra point-response-function (PRF) and convolve the band-corrected ACIS-S image with the appropriate EHRI or RHRI PRF models. In our preliminary investigations we found that the parameters of the analytical PRF models (EHRI: Henry \& Henriksen 1986; RHRI David et al. 1998) needed to be modified slightly from their published values in order to obtain good fits to the E0102.2-7219 data. For both datasets it was the largest spatial scale component of the PRF model that needed adjustment. For the RHRI the best-fit normalization of the $\sim 30^{\prime \prime}$-scale exponential component was 33\% higher than nominal and for the EHRI the normalization of the $\sim 13^{\prime \prime}$-scale power-law component was less than nominal by $22 \%$. E0102.2-7219 is small enough that spatial variations in the background, vignetting, or PRF can all be safely neglected.

The final piece of information required for the expansion study is the plate scales of the three instruments. The plate scales of the EHRI and RHRI are 0".4965 \pm 0 ".0012 per pixel and 0".499 \pm 0 ".001 per pixel, respectively (David et al. 1998). For the ACIS-S the plate scale near the center of the field of view is 0 ". $49115 \pm 0$ ".00010 per pixel based on Chandra in-flight observations of the star cluster NGC 2516 (M. Markevitch 1999, private communication). This value is within $0.2 \%$ of the pre-flight estimate of 0 ". 4920 per pixel.

The approach we follow for determining the expansion rate is that described in Hughes (1999). In brief each HRI image (the "data") is compared to the appropriate band-corrected ACIS-S image (the "model") in a fit for parameters using a maximum-likelihood estimator as the figure-of-merit function. The fitted parameters are the relative intensity scale between the two images, the relative pixel position, the fractional amount of expansion or contraction of the spatial scale, and the normalization of the largest-scale PRF component. The difference in background levels (e.g., ACIS-S vs. EHRI or HRI) is fixed at the value determined from source-free portions of the image and is included as a spatially uniform term. In the fitting software the expansion or contraction of the "model" image is done first using a rebinning scheme that conserves flux, then the scaled image is convolved with the appropriate instrumental PRF, and finally the background term is included. The only interesting parameter that we obtain from this comparison is the expansion (or contraction) factor, which tells us the global mean expansion rate of the remnant. This procedure explicitly assumes that the fractional expansion rate is uniform over the entire remnant, both radially and azimuthally. 
Figure 1 shows the fitted results. E0102.2-7219 has expanded by $0.74 \pm 0.31 \%$ based on comparing the RHRI and ACIS-S data and it has expanded by $1.98 \pm 0.64 \%$ based on comparing the EHRI and ACIS-S data. The quoted errors are purely statistical at 1 sigma and include both Poisson error (dominated by the EHRI and RHRI data) as well as the uncertainty in the plate scales. The two points are described best by a uniform expansion rate of $0.100 \pm 0.025 \% \mathrm{yr}^{-1}$. The null hypothesis that the remnant has remained constant in size can be rejected at the $4 \sigma$ confidence level.

It is extremely unlikely that systematic effects could account for these results. For example, the Chandra plate scale would have to be incorrect by more than $1 \%$, which is 50 times the statistical uncertainty on the calibration measurement. We investigated systematic errors due to uncertainty in the analytical PRF models and in the effective area functions and the boxes plotted in fig. 1 show an estimate of their likely effect on the expansion measurements. Neither is expected to be a dominant source of error.

\section{POST-SHOCK ELECTRON TEMPERATURE}

The eastern limb of E0102.2-7219 in the Chandra image (Blair et al. 2000, Gaetz et al. 2000) displays a smooth, nearly circular, faint rim of X-ray emission. Beyond the edge of the rim the surface brightness drops by more than two orders of magnitude to the average background level over a distance of order $1^{\prime \prime}$. Herein we identify this jump as the SN shock wave plowing into the ambient medium (hereafter "blast wave").

We extracted the spectrum of the blast wave region from within a partial annulus of thickness $1^{\prime \prime} .5$ and outer radius $21^{\prime \prime}$ that covered the angular range from $-30^{\circ}$ to $155^{\circ}$ (angles measured counterclockwise from north). We ignored the background since it was negligibly small (less than 1\%). The spectral data were contained within a single readout node of chip S3, nevertheless the spectrum included data from several detector regions with potentially different intrinsic responses to incident $\mathrm{X}$-rays. Individual response functions (both effective area functions and spectral redistribution matrices) were generated for the several regions covered by the extraction region and then combined, weighting by the number of X-ray events, into a single global response function. The results we obtain are insensitive to this procedure: our fitted parameters are nearly unchanged if we use the response functions from any individual extraction region. Because of the limited statistical precision of our spectrum (which contained only 1500 detected events) the uncertainty on derived parameters is dominated by Poisson counting errors rather than calibration uncertainties.

Figure 2 plots the blast wave spectrum and best-fit nonequilibrium ionization model (NEI) (Hughes \& Singh 1994). The spectrum shows a well-resolved O VIII Ly $\alpha$ line at $0.653 \mathrm{keV}$, a blend of Ne IX and Ne X K-shell lines in addition to Fe L-shell lines around $1 \mathrm{keV}$, and the $\mathrm{Mg}$ XI He-like $2 \rightarrow 1$ line complex at $\sim 1.34 \mathrm{keV}$. The mere presence of these lines suggests a moderately low 
electron temperature, $k T \sim 1 \mathrm{keV}$, as our detailed fits confirm. Table 2 gives best-fit parameters and 1-sigma statistical errors determined from two classes of NEI models: (1) a "single ionization timescale" model which assumes a single, constant value for both the temperature and ionization timescale in the blast wave region and (2) a "planar shock" model which also assumes a constant temperature, but integrates the emission from spectral components with ionization timescales varying linearly from 0 to $n_{e} t$ (the fit parameter) and weighted by equal fractional intervals in $n_{e} t$. The first model provides a marginally acceptable fit to the Chandra data (the $\chi^{2}$ of 57 for 38 degrees of freedom can be rejected at the $97.5 \%$ confidence level). The second model, with no additional parameters, yields a much improved, and statistically acceptable, fit.

Elemental abundances were determined only for the species with obvious line emission in the observed spectrum, viz. O, Ne, Mg, and Fe. The abundances of the other astrophysically common species were fixed at values appropriate to interstellar gas in the SMC (Russell \& Dopita 1992), an assumption that resulted in a significantly better fit than one where the abundances of the other species were fixed to standard solar abundances. The line-of-sight absorbing column density, $N_{\mathrm{H}}$, was separated into a component with normal solar composition arising from the Galaxy and a component from the SMC with lower abundances. The Galactic component was fixed at an $N_{\mathrm{H}}$ value consistent with reddening estimates and the Galactic H I column to E0102.2-7219, while the SMC component was allowed to vary freely.

The abundances from both model fits are nicely consistent with the well-known low metallicity of the gas in the SMC ( $\sim 25 \%$ solar $)$, strongly suggesting that the emission we see from the outer rim comes from the shock wave propagating through interstellar gas. [5 Further in from the blast wave where the X-ray emission of E0102.2-7219 has become quite bright, the spectral character has changed as well. In fig. 2 the spectrum from the bright region in the southeast quadrant is plotted (labeled "ejecta"). The superposed model spectrum, shown for comparison, is the blast wave NEI model scaled in intensity to fit the continuum level of the ejecta spectrum. There are clear differences between the ejecta and blast wave spectra in terms of both thermodynamic state (note, for example, the strength of the O VII He-like $2 \rightarrow 1$ line complex at $\sim 0.57 \mathrm{keV}$ in the ejecta which is virtually absent in the blast wave) and the elemental abundances (especially of $\mathrm{O}$ and $\mathrm{Ne}$ ). The enhanced abundances and less advanced ionization state of the ejecta are consistent with expectations for emission from the reverse shock propagating into higher density, metal-rich gas.

\footnotetext{
${ }^{3}$ One should be wary of interpreting the fitted abundances much beyond this. In particular, the Fe abundance comes from a complex of L-shell lines that are unresolved at the modest spectral resolution of our data. Current models (including ours) are known to be incomplete as regards the Fe L-shell emission (Brickhouse et al. 2000), which introduces additional uncertainty beyond the statistical errors in the measured value of the Fe-abundance.
} 


\section{DISCUSSION}

The X-ray expansion rate of E0102.2-7219 is fully consistent with the remnant's proper motion estimated from the location and radial velocities of the oxygen-rich optical filaments. Specifically our estimate of the remnant's age assuming free expansion, $1000_{-200}^{+340} \mathrm{yr}$, agrees with that of Tuohy \& Dopita (1983). At the outermost edge of the X-ray emission from E0102.2-7219 $\left(R \approx 22^{\prime \prime}=6.4 \mathrm{pc}\right)$, the inferred velocity is $6200_{-1600}^{+1500} \mathrm{~km} \mathrm{~s}^{-1}$, which we consider in the following to be the speed of the blast wave. According to the Rankine-Huguniot jump conditions in the absence of cosmic ray acceleration (Landau \& Lifshitz 1976), this velocity corresponds to a mean post-shock temperature of $k T_{S}=\frac{3}{16} \mu m_{p} v_{S}^{2}=45_{-20}^{+25} \mathrm{keV}$, using a mean mass per particle of $\mu=0.61$. Our measurement of the post-shock electron temperature from the Chandra X-ray spectrum, $k T \lesssim 1 \mathrm{keV}$, is at least 25 times smaller than this estimate.

There are two principle assumptions that go into our estimate for the mean temperature: (1) that the post-shock thermal energy is partitioned equally between electrons and ions, and (2) that only a negligible fraction of the total energy of the shock goes into cosmic rays.

It has been debated whether electron and ion temperatures are quickly equilibrated by plasma processes behind collisionless high Mach number SN shocks (McKee 1974; Cargill \& Papadopoulos 1988 ) or if the electron and ion temperatures initially differ by their mass ratio $\left(m_{p} / m_{e}=1836\right)$ and then equilibrate slowly through Coulomb collisions (Shklovskii 1968). For E0102.2-7219 we find, ignoring for the moment any Coulombic heating of the electrons downstream, that the ratio of electron thermal energy to upstream kinetic energy, $E_{\mathrm{e}, \mathrm{th}} / E_{\mathrm{kin}}=k T_{e} /\left(\frac{1}{2} m_{p} v_{S}^{2}\right) \lesssim 1 \%$, is much smaller than the value of $12 \%$ expected by Cargill \& Papadopoulos (1988).

Downstream of the blast wave, electrons and ions (mainly protons) will exchange energy through Coulomb interactions, which provide the minimum level of heating expected in the case of nonequipartition. The electron temperature varies as

$$
\frac{d T_{e}}{d n_{e} t}=0.13 \frac{T_{p}-T_{e}}{T_{e}^{3 / 2}}
$$

(in cgs units) (Spitzer 1978, p. 22), where we express the temperature variation in terms of $n_{e} t$, since we have an observational estimate of this quantity from the X-ray spectral fits (Table 2). This equation predicts that the mean post-shock electron temperature (averaging over $n_{e} t$ from 0 to $4 \times 10^{11} \mathrm{~cm}^{-3} \mathrm{~s}$ ) would be between $4.5 \mathrm{keV}$ and $8 \mathrm{keV}$ for proton temperatures corresponding to the measured shock velocity (i.e., $T_{p}=40-120 \mathrm{keV}$ ). Note that the dependence of electron temperature on the actual value of $n_{e} t$ up to which the averaging is done is weak. If the lower limit on the measured $n_{e} t$ value, $2 \times 10^{11} \mathrm{~cm}^{-3} \mathrm{~s}$, from our spectral fits is used, then the average $k T_{e}$ range becomes $3.5-6 \mathrm{keV}$. This estimate assumes that the total pressure $\left(n_{p} T_{p}+n_{e} T_{e}\right)$ remains constant in the post-shock region, when, in fact, some adiabatic decompression should occur as the shocked gas elements expand outward. Estimates of this effect, assuming the post-shock gas 
acquires a speed of $3 / 4 v_{S}$, indicate that the predicted electron temperatures may be reduced to no lower than $\sim 2.5 \mathrm{keV}$, closer to, but still considerably higher than, the measured blast wave electron temperature in E0102.2-7219. These simple models suggest that post-shock proton temperatures of $40-120 \mathrm{keV}$ are just too high and that they need to be considerably reduced in order to match the Chandra measurements. This in turn argues that cosmic ray production is not negligible in E0102.2-7219 and that the relativistic particles may be absorbing a significant fraction of the SN shock energy.

Recently Decourchelle et al. (2000) have shown that the fraction of energy going into cosmic rays is likely to be high in young SNRs and that this effect has direct consequences for the thermal X-ray emission. In such a situation, the shock jump conditions are modified (e.g., Blandford \& Eichler 1987) so that for a given shock velocity the compression ratio increases and the post-shock temperature decreases. Nonlinear models of shock acceleration (Ellison 2000) appear fully consistent with the observed shock parameters of E0102.2-7219. In particular, these models predict a mean post-shock temperature of $1 \mathrm{keV}$ (see figure 1 in Ellison 2000) for standard cosmic ray injection efficiencies and a high Mach number shock, i.e., values of 100-300, as appropriate for E0102.2-7219. Thus it appears that efficient cosmic ray acceleration alone is sufficient to explain our observations of E0102.2-7219, even without the additional influence of nonequipartition of electron and ion temperatures.

Unfortunately, a direct measurement of the post-shock proton temperature in E0102.2-7219 is not possible, due to the absence of any $\mathrm{H}$ line emission from the remnant. Therefore, constraints on the efficiency of cosmic ray production in E0102.2-7219 will need to come from future detailed studies of the compression ratio, the structure of the forward and reverse shocks, and the X-ray line spectrum using NEI hydrodynamical models of SNRs that include cosmic-ray acceleration (e.g., Decourchelle et al. 2000). In addition, studies of other types of remnants, in particular Balmer-dominated ones for which estimates of the proton temperature are available from $\mathrm{H} \alpha$ line profiles, should prove helpful in further elucidating the physics of collisionless shock fronts.

Helpful discussions with David Burrows, Don Ellison, Maxim Markevitch, Jon Morse, and Wallace Tucker on the scientific content of the article are gratefully acknowledged. We appreciate Monique Arnaud's support and hospitality during the course of this project. This work was partially supported by NASA Grant NAG5-6420. 


\section{REFERENCES}

Blair, W. P., et al. 2000, ApJ, in press

Blandford, R. D. \& Eichler, D. 1987, Phys. Reports, 154, 1

Brickhouse, N. S., Dupree, A. K., Edgar, R. J., Liedahl, D. A., Drake, S. A., White, N. E., \& Singh, K. P. 2000, ApJ, 530, 387

Cargill, P. J., \& Papadopoulos, K. 1988, ApJ, 329, L29

Chevalier, R. A. 1983, ApJ, 272, 765

David, L. P., et al. 1998, The ROSAT High Resolution Imager (HRI) Calibration Report (http://hea-www.harvard.edu/rosat/rsdc-www/hricalrep.htm])

Decourchelle A., Ellison, D. C., \& Ballet, J. 2000, ApJL, submitted.

Dewey, D. 1999, PSF Studies...Encircled Energy Curves: Expected and Measured, AXAF Science Center Calibration memo from 8/19/99

Dopita, M. A., Tuohy, I. R., \& Mathewson, D. S. 1981, ApJ, 248, L105

Ellison, D. C. 2000, in AIP Conf. Proc. 528, Acceleration and Transport of Energetic Particles Observed in the Heliosphere, ed. R. A. Mewaldt, J. R. Jokipii, M. A. Lee, E. Moebius, \& T. H. Zurbuchen, in press astro-ph/0003214

Gaetz, T. J., Butt, Y. M., Edgar, R. J., Eriksen, K. A., Plucinsky, P. P., Schlegel, E. M., \& Smith R. K. 2000, ApJ, 534, L47

Garmire, G. P. 1997, AAS Meeting, 190, 34.04

Giacconi, R., et al. 1979, ApJ, 230, 540

Hayashi, I., Koyama, K., Ozaki, M., Miyata, E., Tsunemi, H., Hughes, J. P., \& Petre, R. 1994, PASJ, 46, L121

Henry, J. P., \& Henriksen, M. J. 1986, ApJ, 301, 689

Hughes, J. P. 1988, in IAU Colloq. 101, Supernova Remnants and the Interstellar Medium, ed. R. S. Rogers \& T. L. Landecker (Cambridge: Cambridge University Press), 125.

Hughes, J. P. 1999, ApJ, 527, 298

Hughes, J. P., \& Singh, K. P. 1994, ApJ, 422, 126

Landau, L. D., \& Lifshitz, E. M. 1976, Fluid Mechanics, (New York: Pergamon Press)

McKee, C. E. 1974, ApJ, 188, 335

Russell, S. C., \& Dopita, M. A. 1992, ApJ, 384, 508

Shklovskii, I. S. 1968, Supernovae (New York: Wiley)

Spitzer, L. 1978, Physical Processes in the Interstellar Medium (New York: Wiley-Interscience)

Tuohy, I. R., \& Dopita, M. A. 1983, ApJ, 268, L11 
van den Bergh, S. 2000, PASP, 112, 529

Weisskopf, M. C., O’Dell, S. L., \& Van Speybroeck, L. P. 1996, Proc. SPIE, 2805, 2

This preprint was prepared with the AAS $\mathrm{LAT}_{\mathrm{E}} \mathrm{X}$ macros v4.0. 
Table 1. Observations of E0102.2-7219

\begin{tabular}{cccc}
\hline \hline Observatory & Start Date & Average MJD & Duration (s) \\
\hline Einstein (EHRI) & 1980 Apr 18 & 44347.5 & 22500 \\
ROSAT (RHRI) & 1991 Nov 9 & 48598.2 & 21900 \\
$A C I S-S$ & 1999 Aug 23 & 51413.9 & 8883 \\
\hline
\end{tabular}

Table 2. NEI Models of E0102.2-0.5

\begin{tabular}{ccc}
\hline \hline & $\begin{array}{c}\text { Single Ionization } \\
\text { Timescale }\end{array}$ & Planar Shock \\
\hline$k T(\mathrm{keV})$ & $0.48_{-0.05}^{+0.08}$ & $0.78_{-0.15}^{+0.16}$ \\
$n_{e} t\left(\mathrm{~cm}^{-3} \mathrm{~s}\right)$ & $4_{-2}^{+21} \times 10^{11}$ & $4_{-2}^{+4} \times 10^{11}$ \\
$N_{\mathrm{H}, \mathrm{SMC}^{\mathrm{a}}\left(\mathrm{cm}^{-2}\right)}(5 \pm 3) \times 10^{20}$ & $<2.5 \times 10^{20}$ \\
$n_{\mathrm{e}} n_{\mathrm{H}} \mathrm{V} / 4 \pi \mathrm{D}^{2}\left(\mathrm{~cm}^{-5}\right)$ & $1.1_{-0.3}^{+0.4} \times 10^{11}$ & $4.1_{-0.8}^{+2.0} \times 10^{10}$ \\
Oxygen & $0.26_{-0.14}^{+0.19}$ & $0.34_{-0.12}^{+0.15}$ \\
Neon & $0.31_{-0.09}^{+0.11}$ & $0.67_{-0.19}^{+0.20}$ \\
Magnesium & $0.28_{-0.10}^{+0.12}$ & $0.47 \pm 0.16$ \\
Iron & $0.01 \pm 0.005$ & $0.06 \pm 0.03$ \\
$\chi^{2}$ (d.o.f.) & $57.13(38)$ & $46.07(38)$ \\
\hline
\end{tabular}

${ }^{a}$ Uses mean SMC abundances; fits also include a fixed $N_{\mathrm{H}, \text { Gal }}$ of $5 \times 10^{20} \mathrm{~cm}^{-2}$ using solar abundances 
Fig. 1.- Global mean percentage expansion of SNR E0102.2-7219 versus the time difference between imaging measurements. The point indicating the shortest time difference comes from comparing the ROSAT and Chandra observations. The other point comes from comparing the Einstein and Chandra observations. The error bars show the statistical uncertainty, while the boxes that surround each data point give an estimate of the systematic uncertainty. The dotted curves show the best-fit percentage expansion and 1 sigma errors. The inferred speed of the shock front is $\sim 6000 \mathrm{~km} \mathrm{~s}^{-1}$ and the remnant's age is $1000 \mathrm{yr}$.

Fig. 2. - Chandra spectra of a portion of the outer blast wave and bright ejecta of SNR E0102.2-7219 as indicated. The spectra were rebinned to a minimum of 25 events per channel. The best-fit nonequilibrium ionization model for the blast wave is shown as the solid histogram. The model appears compared against the ejecta spectrum as well in order to demonstrate the gross spectral differences between the two regions. The residuals are shown only for the blast wave spectrum. 


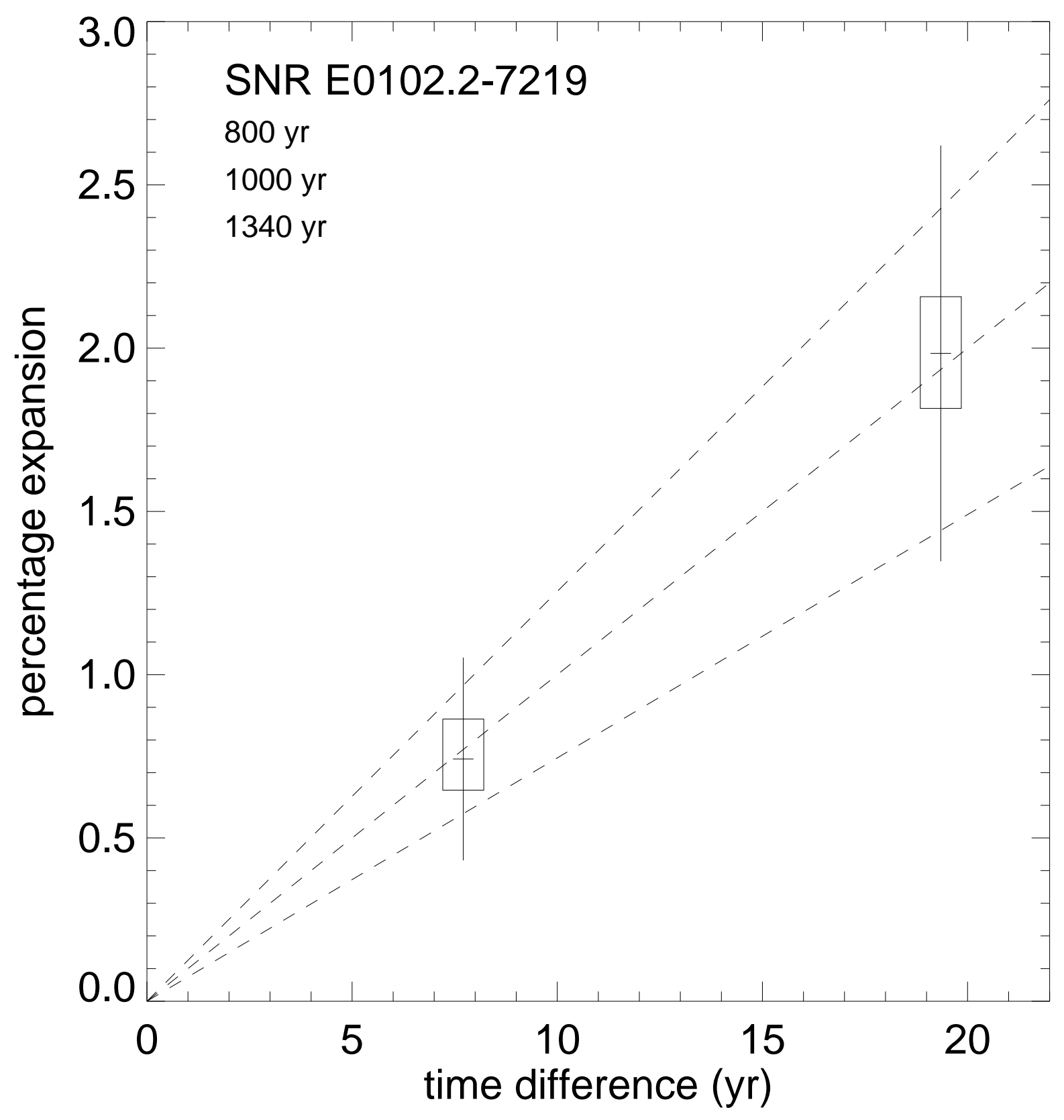




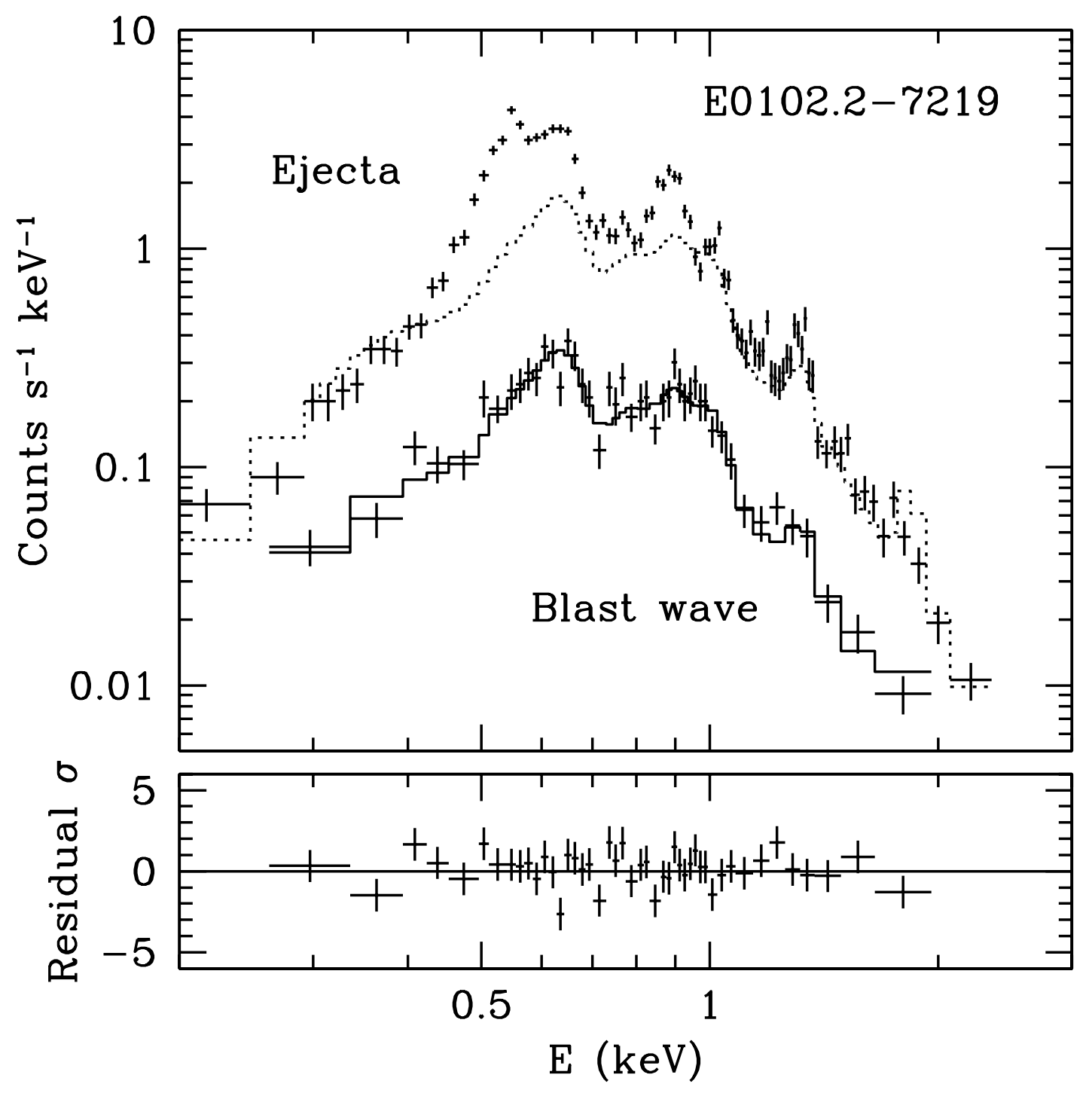

